\title{
The Volume as a Metric Invariant of Polyhedra*
}

\author{
I. Kh. Sabitov \\ Faculty of Mechanics and Mathematics, Moscow State University, \\ Moscow, 119899 Russia \\ matan@nss.msu.su
}

\begin{abstract}
It is proved that in $R^{3}$ the volume of any polyhedron is a root of some polynomial with coefficients depending only on the combinatorial structure and the metric of the polyhedron. As a consequence, we have a proof of the "bellows conjecture" affirming the invariance of the volume of a flexible polyhedron in the process of its flexion.
\end{abstract}

\section{Introduction}

Let $P$ be a polyhedral surface in $R^{3}$. A continuous deformation of $P$ is called a flexion if it changes only (some or all of) dihedral angles of $P$ and keeps each face congruent to itself. The first example of a flexible polyhedron embedded in $R^{3}$ (i.e., without selfintersections) was found by Connelly [6]. Shortly after that it was noticed that the volume of Connelly's flexing polyhedron, as well as of others constructed later, does not change during their flexion and therefore Connelly [7] suggested that this property is common for all flexing polyhedra. Since then his conjecture (named for obvious reasons the "bellows conjecture") has been considered to be one of the most interesting problems in flexion theory.

The first attempt to solve this problem was made by Sabitov [12] and it was based on the approach proposed in [9] and [11]. The prospects of this approach were shown first by Pavlova [10] for the case of some simple polyhedra. At present two proofs of the bellows conjecture are known [8], [13]; both are based on the same geometric idea as the one in [12], but use different algebraic tools for its realization. Here we give an extended English version of [13] with some modifications in the presentation of the proof.

The article is organized as follows. In Section 2 we introduce some necessary definitions. In Section 3 we explain our approach to the bellows conjecture (this section

\footnotetext{
* This work was supported by RFFI (Russia) Grant No. 96-01-00836.
} 
may be omitted without loss of understanding of the following text). The main result and its corollaries are formulated in Section 4. Proofs are given in Section 5-8. Finally, in Section 9 we address some applications of the results.

\section{Polyhedra in $\boldsymbol{R}^{3}$ and Their Generalized Volume}

Given a simplicial 2-complex $K$, a polyhedron (or a polyhedral surface) in $R^{3}$ with combinatorial structure $K$ is defined as a continuous map $P:|K| \rightarrow R^{3}$, where $|K|$ is the body (carrier) of $K$. Of course, the map $P$ is supposed to be linear on each simplex of $K$. We consider only the case when $|K|$ is homeomorphic to an orientable 2-manifold of genus $g \geq 0$. Often by a polyhedron we also mean the image $P(|K|) \subset R^{3}$ rather than the map $P$. That image will sometimes also be denoted by $P$ when it causes no confusion.

Note that the map $P$ is not required to be injective on $|K|$, or even on a single simplex of $K$, which means that the geometric polyhedron $P$ may have degenerate faces, selfintersections, or even self-superpositions. To define the volume of a polyhedron in these singular cases, we use the concept of generalized volume. The polyhedron $P$ with the orientation naturally inherited from $K$ is called an oriented polyhedron. Choose a point $O$ in $R^{3}$ and compose the sum $\sum V_{i}$ of coherently oriented volumes of all the tetrahedra spanned by the point $O$ and the faces of $P$.

Definition. $\quad V=\sum V_{i}$ is called the generalized oriented volume of $P$.

Evidently, the value of generalized volume is independent of the choice of point $O$. It is also clear that for any embedded polyhedron its usual oriented volume and its generalized volume actually coincide.

Recall that the oriented volume of a tetrahedron with vertices $O, M_{1}, M_{2}, M_{3}$ is equal to one-sixth of the mixed product of vectors $O M_{i}, i=1,2,3$, so the generalized volume $V(P)$ is a polynomial in coordinates of the vertices of $P$.

\section{Motivations for Our Approach to the Problem}

\subsection{Volumes of Some Simple Polyhedra}

The Volume of a Tetrahedron. It is well known that the volume $V$ of a tetrahedron (see Fig. 1) with given lengths of the edges $l_{1}, l_{2}, l_{3}, l_{4}, l_{5}, l_{6}$ may be calculated by the following formula:

$$
\begin{gathered}
V^{2}=\frac{1}{144}\left[l_{1}^{2} l_{5}^{2}\left(l_{2}^{2}+l_{3}^{2}+l_{4}^{2}+l_{6}^{2}-l_{1}^{2}-l_{5}^{2}\right)+l_{2}^{2} l_{6}^{2}\left(l_{1}^{2}+l_{3}^{2}+l_{4}^{2}+l_{5}^{2}-l_{2}^{2}-l_{6}^{2}\right)\right. \\
\left.+l_{3}^{2} l_{4}^{2}\left(l_{1}^{2}+l_{2}^{2}+l_{5}^{2}+l_{6}^{2}-l_{3}^{2}-l_{4}^{2}\right)-l_{1}^{2} l_{2}^{2} l_{4}^{2}-l_{2}^{2} l_{3}^{2} l_{5}^{2}-l_{1}^{2} l_{3}^{2} l_{6}^{2}-l_{4}^{2} l_{5}^{2} l_{6}^{2}\right] .
\end{gathered}
$$

Thus the oriented volume $V$ of any tetrahedron may be regarded as a root of a polynomial equation of the form

$$
Q(V)=V^{2}+a(l)=0,
$$




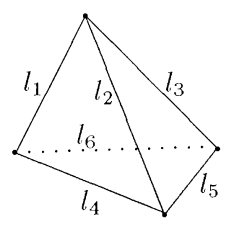

Fig. 1

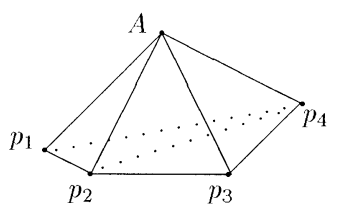

Fig. 2

where $l=\left(l_{1}^{2}, \ldots, l_{6}^{2}\right)$ denotes the set of the squares of the lengths of the edges, and $a(l)$ is a polynomial in $l$ with rational coefficients.

The Generalized Volume of a Polyhedron with Five Vertices. It is easy to show that such a polyhedron $P$ always has three vertices of degree 4 and two vertices of degree 3. So the combinatorial structure of $P$ is determined uniquely and is shown in Fig. 2, where the base of the quadrangular pyramid is supposed to be dissected by one of its diagonals. Decomposing $P$ into two tetrahedra, $\left\langle A p_{1} p_{2} p_{4}\right\rangle$ and $\left\langle A p_{2} p_{3} p_{4}\right\rangle$, we have $V=\operatorname{vol}(P)=V_{A 124}+\varepsilon V_{A 234}, \varepsilon= \pm 1$. Hence $V^{4}-2\left(V_{1}^{2}+V_{2}^{2}\right) V^{2}+\left(V_{1}^{2}-V_{2}^{2}\right)^{2}=0$, where $V_{1}=V_{A 124}, V_{2}=V_{A 234}$. Replacing the values of $V_{1}^{2}$ and $V_{2}^{2}$ by their expressions in the form of (1), we obtain that the volume of any polyhedron with five vertices is a root of a polynomial equation of the form

$$
Q(V)=V^{4}+a_{1}(l) V^{2}+a_{2}(l)=0,
$$

where $l$ denotes the set of the squares of the edge lengths of the polyhedron, and $a_{1}, a_{2}$ are some polynomials in $l$ with rational coefficients.

We would like to emphasize that (2) is valid for all polyhedra with five vertices independent of their actual configuration in $R^{3}$. For example, consider some possible cases:

(1) $V_{1}=V_{2} \neq 0$. Then we have $Q(V)=V^{4}-4 V_{1}^{2} V^{2}=0$. Therefore, $V= \pm 2 V_{1}$ in the case where vertices $p_{1}$ and $p_{3}$ are separated by the plane passing through vertices $p_{2}, p_{4}$, and $A$; and $V=0$ in the case where vertices $p_{1}$ and $p_{3}$ are not separated by that plane. In both cases the volume of $P$ is a root of the same equation (2).

(2) $V_{2}=0, V_{1} \neq 0$ (in Fig. 3 vertex $p_{3}$ is in the plane $\left\langle A p_{2} p_{4}\right\rangle$ ). Then $Q(V)=$ $\left(V^{2}-V_{1}^{2}\right)^{2}=0$, and hence $V= \pm V_{1}$.

(3) The lengths $l_{A 2}=l_{A 4}, l_{24}=0$. Then $V_{1}=0, V_{2}=0$. Hence $Q(V)=V^{4}=0$, and so $V=0$. By the way, in this case the polyhedron $P$ (see Fig. 4) admits

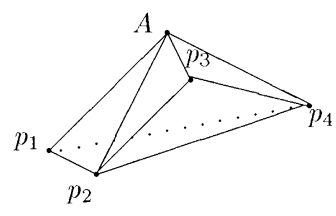

Fig. 3

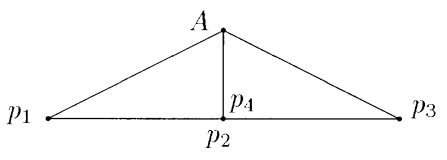

Fig. 4 
nontrivial flexions, namely, the rotation of the three faces $\left\langle A p_{2} p_{3}\right\rangle,\left\langle A p_{3} p_{4}\right\rangle$, and $\left\langle p_{2} p_{3} p_{4}\right\rangle$ (this one is a degenerate face) about the axis $\left\langle A p_{2}\right\rangle=\left\langle A p_{4}\right\rangle$; however, the value of volume is always a root of the same equation $Q(V)=V^{4}=0$.

By the above argument, we deduce the first observation: in the simplest cases the volume of polyhedra satisfies a polynomial equation with coefficients independent of the positions of vertices and polynomially dependent only on the squares of the lengths of the edges.

\subsection{Polyhedra as Algebraic Varieties}

As we know from Section 2, a polyhedron in $R^{3}$ is defined as a map $P: K \rightarrow R^{3}$, where $|K|$ is the carrier of a simplicial complex $K$. Since all faces of $K$ are triangles and $P$ is linear on each face, it follows that the map $P$ is completely determined by the images $M_{i}\left(x_{i}, y_{i}, z_{i}\right), 1 \leq i \leq n$, of $n$ vertices of $K$. Therefore, for each polyhedron $P$ in $R^{3}$, we can assign a point $M\left(x_{1}, y_{1}, z_{1}, \ldots, x_{n}, y_{n}, z_{n}\right) \in R^{3 n}$ and, conversely, with any point $M \in R^{3 n}$ we can obviously associate a polyhedron $P$ in $R^{3}$ so that there is a bijective (even homeomorphic in a corresponding topology) relation $R^{3} \supset P \leftrightarrow M \in R^{3 n}$.

The number of edges of $P$ is $e=3 n-6+6 g$, where $g \geq 0$ is the topological genus of the polyhedron $P$. Under a given numbering of the vertices of $P$, assume that the edges are numbered by the index $k=k(i, j), 1 \leq k \leq e$, where $i$ and $j$ are numbers of the vertices joined by the edge $k$. The lengths of edges are given by the following equations:

$$
\left(x_{i}-x_{j}\right)^{2}+\left(y_{i}-y_{j}\right)^{2}+\left(z_{i}-z_{j}\right)^{2}=l_{k}^{2}, \quad 1 \leq k \leq e .
$$

Now we consider all solutions $\left(x_{1}, \ldots, z_{n}\right)$ of (3) with the restriction that the lengths $l_{k}$ are fixed. Thus we obtain all polyhedra in $R^{3}$ with rigid faces isometric to $P$, and having the same combinatorial structure $K$. In order to exclude the polyhedra obtained by a parallel translation of $P$, we add three equations to (3):

$$
\sum_{i} x_{i}=0, \quad \sum_{i} y_{i}=0, \quad \sum_{i} z_{i}=0 .
$$

It is easy to show that the solutions of (3)-(4) are situated in $R^{3 n}$ in a ball $B \subset R^{3 n}$ of a finite radius $r$ depending on the combinatorial structure $K$ and on $l=\left(l_{1}^{2}, \ldots, l_{e}^{2}\right)$, the squares of the lengths of the edges. So the set $\tilde{P}$ of polyhedra in $R^{3}$ isometric to $P$ and having the same combinatorial structure $K$ is in a homeomorphic relation with the algebraic variety $\tilde{A}$ defined by system (3)-(4). However, $\tilde{A}$ in the ball $B$ may have only a finite number of compact components (which may have different dimensions). If we add three new equations to (3)-(4) excluding continuous rotations of $P$, then any one-point component of $\tilde{A}$ corresponds to a rigid polyhedron in $\tilde{P}$ and the other ones are composed by flexing polyhedra in $\tilde{P}$. Hence we have come to the second observation: if the bellows conjecture if true, then the volumes of all polyhedra in $\tilde{P}$ have only a finite number of possible values. 


\section{The Main Result}

Starting from these observations, we have suggested (see, for example, Section 8 in [9] by the author) that instead of seeking a proof of the bellows conjecture considering only flexible polyhedra, it would be more hopeful to try proving a more general conjecture, namely, the volume of any polyhedron $P$ in $R^{3}$ is a root of some polynomial equation $Q(V)=0$ with coefficients depending only on its metric and combinatorial structure. In fact, we prove the following theorem:

Theorem 1. Let $P$ be an oriented polyhedral surface in $R^{3}$ having a given combinatorial structure $K$ and given values of edge lengths $l_{k}, 1 \leq k \leq e$, where $e$ is the number of edges of $P$. Let $\tilde{P}$ be the set of all polyhedra in $R^{3}$ with the same combinatorial structure $K$ and having the same lengths of the edges as $P$. Then there exists a polynomial equation

$$
Q(V)=V^{2 N}+a_{1}(l) V^{2 N-2}+\cdots+a_{N}(l)=0
$$

such that the generalized volume of any polyhedron from $\tilde{P}$ is a root of this equation. Moreover, the coefficients $a_{i}$ are polynomials in $(l)=\left(l_{1}^{2}, \ldots, l_{e}^{2}\right)$ with rational coefficients depending on $K$.

Corollary 1. For all polyhedra isometric to a given polyhedron, there is only a finite number of possible values of their volumes.

Thus we can say that the volume of a polyhedron is a finitely valued function of the polyhedron's metric, and therefore it is natural to interpret the theorem as a generalization of Heron's formula for the area of a triangle.

Corollary 2. The bellows conjecture is true.

Indeed, the volume $V$ of a flexible polyhedron $P$ is a continuous function of positions of its vertices. However, by Corollary 1 , in the process of flexion of $P, V$ can take only a finite number of values, so that $V$ remains constant.

Remarks. (1) The algebraic meaning of the theorem is as follows. Denote by $\psi_{k}$ $\left(x_{1}, \ldots, z_{n}\right), 1 \leq k \leq e$, the polynomials in the left-hand side of (3). The volume $V(P)$ is a polynomial over the same variables $\left(x_{1}, \ldots, z_{n}\right)$. The theorem asserts that this polynomial $V\left(x_{1}, \ldots, z_{n}\right)$ algebraically depends on the polynomials $\psi_{k}$, that is, there is a polynomial $Q\left(V, \psi_{1}, \ldots, \psi_{e}\right)$ such that $Q\left(V\left(x_{1}, \ldots, z_{n}\right), \psi_{1}\left(x_{1}, \ldots, z_{n}\right), \ldots\right.$, $\left.\psi_{e}\left(x_{1}, \ldots, z_{n}\right)\right) \equiv 0$ over the ring $R\left(x_{1}, \ldots, z_{n}\right)$. It seems that the existence of a polynomial identity for $V$ and $\psi_{k}$ is not very difficult to establish as is pointed out in [12]; however, in general all the coefficients for $V$ in $Q$ may be equal to zero for some values of $(l)$. In [13] we showed the existence (over the ring of rational numbers) of such a polynomial $Q(V)$ with the leader coefficient 1 . After this in [8], with help of places' 
theory, the existence of such a polynomial over the ring of integers whose leading coefficient is also 1 if the independent variable in $Q$ is taken equal to $12 \mathrm{~V}$ is established. For our polynomial $Q(V)$ in (5) we know not only the existence but a method for its construction too. The numerical coefficients in $a_{i}(l)$ are rational numbers, but in fact one could trace out that for $\tilde{V}=12 \mathrm{~V}$ the coefficients of the corresponding polynomial are integral; in particular, the leading coefficient is 1, exactly as in [8].

(2) Our method of constructing a required polynomial gives many such polynomials. However, excluding the case of octahedra [3], we know neither the minimum degree of these equations nor their canonical form. Of course, using our method, we can construct all possible polynomials $Q(V)$ and take their greatest common divisor over the ring $R\left[l_{1}^{2}, \ldots, l_{e}^{2}\right]$ (may be even over $R\left[l_{1}, \ldots, l_{e}\right]$ ), but we do not know if it is a required polynomial of minimum degree.

(3) The theorem is true also for polyhedra with nontriangular rigid faces because we can decompose them into triangles by adding diagonals.

(4) Alexandrov has constructed [2] the example of a flexible polyhedron in threedimensional spherical space $S^{3}$ for which the volume does change under flexion. Therefore the property that the volume may be a function of the polyhedron's metric is not stable as for the curvature's variation of the ambient Euclidean space because in Alexandrov's example the curvature of $S^{3}$ may be chosen arbitrarily close to zero.

(5) It is known that apart from the volume $V$ of a polyhedron, there exists one more geometric magnitude, namely, the mean curvature $H$ of a polyhedron, which also conserves its value during flexion [1]. By definition, $H=\sum_{k} l_{k}\left(\pi-\varphi_{k}\right)$, where $l_{k}$ are the edge lengths, $\varphi_{k}$ are dihedral angles at the corresponding edges, and the sum is taken over all the edges of polyderon. It is noticed in Section 9 that if we know a value of $V$ in general, we can find only a finite number of polyhedra having this volume (of course, we suppose the combinatorial structure and metric of polyhedra to be known and fixed) and consequently a finite number of possible values of $H$. Then the following questions arise: (a) What kind of relation $f(V, H)=0$ exists between $V$ and $H$; in particular, is it polynomial or not? (b) Given some consistent values of $V$ and $H$, do they define the corresponding polyhedron uniquely or not?

\section{The Cayley-Menger Equation}

It is known that ten distances between five points in $R^{3}$ cannot be chosen arbitrarily but must satisfy the Cayley-Menger equation, see, for example, [4] or [5]. Denote by $l$ with two subscripts the distance between the corresponding points, see Fig. 5 on which the complete graph of five points is drawn. Then the Cayley-Menger equation may be

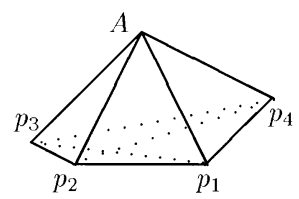

Fig. 5 
written in the form

$$
\left|\begin{array}{cccccc}
0 & 1 & 1 & 1 & 1 & 1 \\
1 & 0 & l_{A 1}^{2} & l_{A 2}^{2} & l_{A 3}^{2} & l_{A 4}^{2} \\
1 & l_{1 A}^{2} & 0 & l_{12}^{2} & l_{13}^{2} & l_{14}^{2} \\
1 & l_{2 A}^{2} & l_{21}^{2} & 0 & l_{23}^{2} & l_{24}^{2} \\
1 & l_{3 A}^{2} & l_{31}^{2} & l_{32}^{2} & 0 & l_{34}^{2} \\
1 & l_{4 A}^{2} & l_{41}^{2} & l_{42}^{2} & l_{43}^{2} & 0
\end{array}\right|=0 .
$$

We aim to present this equation in a suitable form. For this, first we calculate the determinant, $\Delta$, on the left-hand side of the equation. We put $x=l_{13}^{2}, y=l_{24}^{2}$ and present the elements of any column containing $x$ and $y$ as the sum of these elements with zero; using the well-known property of the determinant having a column-sum we obtain

$$
\begin{aligned}
& \Delta=\left|\begin{array}{cccccc}
0 & 1 & 1+0 & 1+0 & 1+0 & 1+0 \\
1 & 0 & l_{A 1}^{2}+0 & l_{A 2}^{2}+0 & l_{A 3}^{2}+0 & l_{A 4}^{2}+0 \\
1 & l_{1 A}^{2} & 0+0 & l_{12}^{2}+0 & 0+x & l_{14}^{2}+0 \\
1 & 1_{2 A}^{2} & l_{21}^{2}+0 & 0+0 & l_{23}^{2}+0 & 0+y \\
1 & l_{3 A}^{2} & 0+x & l_{32}^{2}+0 & 0+0 & l_{34}^{2}+0 \\
1 & l_{4 A}^{2} & l_{41}^{2}+0 & 0+y & l_{43}^{2}+0 & 0+0
\end{array}\right|
\end{aligned}
$$

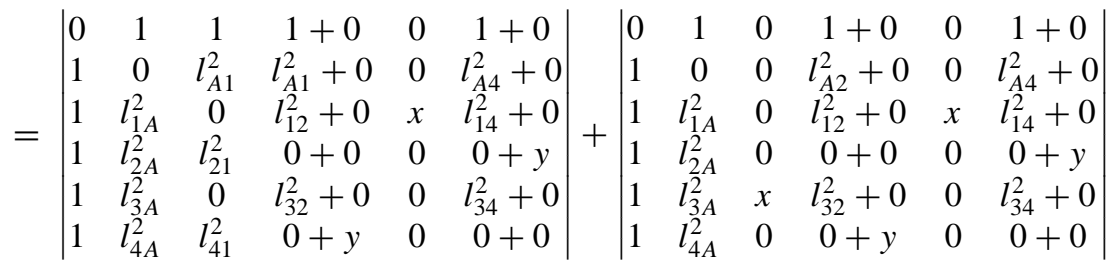

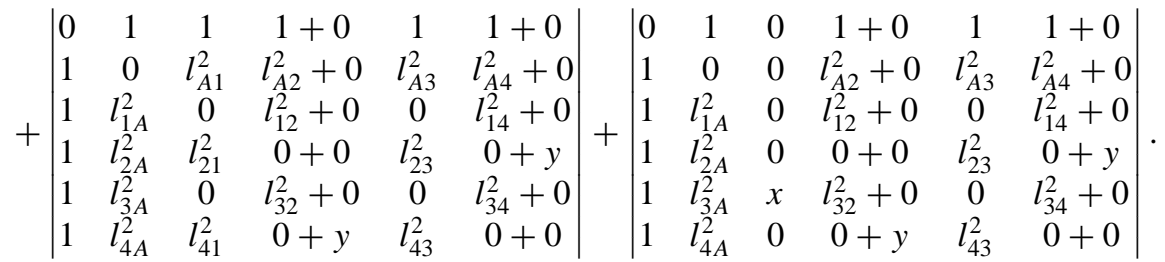

Performing this procedure for the columns containing the sums $0+y$ we present $\Delta$ as the sum of 16 determinants:

$$
\begin{aligned}
& \Delta=\left|\begin{array}{cccccc}
0 & 1 & 1 & 1 & 0 & 1 \\
1 & 0 & l_{A 1}^{2} & l_{A 2}^{2} & 0 & l_{A 4}^{2} \\
1 & l_{1 A}^{2} & 0 & l_{12}^{2} & x & l_{14}^{2} \\
1 & l_{2 A}^{2} & l_{21}^{2} & 0 & 0 & 0 \\
1 & l_{3 A}^{2} & 0 & l_{32}^{2} & 0 & l_{34}^{2} \\
1 & l_{4 A}^{2} & l_{41}^{2} & y & 0 & 0
\end{array}\right|+\left|\begin{array}{cccccc}
0 & 1 & 1 & 0 & 0 & 1 \\
1 & 0 & l_{A 1}^{2} & 0 & 0 & l_{A 4}^{2} \\
1 & l_{1 A}^{2} & 0 & 0 & x & l_{14}^{2} \\
1 & l_{2 A}^{2} & l_{21}^{2} & 0 & 0 & 0 \\
1 & l_{3 A}^{2} & 0 & 0 & 0 & l_{34}^{2} \\
1 & l_{4 A}^{2} & l_{41}^{2} & 0 & 0 & 0
\end{array}\right| \\
& +\left|\begin{array}{cccccc}
0 & 1 & 1 & 1 & 0 & 0 \\
1 & 0 & l_{A 1}^{2} & l_{A 2}^{2} & 0 & 0 \\
1 & l_{1 A}^{2} & 0 & l_{12}^{2} & x & 0 \\
1 & l_{2 A}^{2} & l_{21}^{2} & 0 & 0 & y \\
1 & l_{3 A}^{2} & 0 & l_{32}^{2} & 0 & 0 \\
1 & l_{4 A}^{2} & l_{41}^{2} & 0 & 0 & 0
\end{array}\right|+\left|\begin{array}{cccccc}
0 & 1 & 1 & 0 & 0 & 0 \\
1 & 0 & l_{A 1}^{2} & 0 & 0 & 0 \\
1 & l_{1 A}^{2} & 0 & 0 & x & 0 \\
1 & l_{2 A}^{2} & l_{21}^{2} & 0 & 0 & y \\
1 & l_{3 A}^{2} & 0 & 0 & 0 & 0 \\
1 & l_{4 A}^{2} & l_{41}^{2} & y & 0 & 0
\end{array}\right|
\end{aligned}
$$




$$
\begin{aligned}
& +\left|\begin{array}{cccccc}
0 & 1 & 0 & 1 & 0 & 0 \\
1 & 0 & 0 & l_{A 2}^{2} & 0 & 0 \\
1 & l_{1 A}^{2} & 0 & l_{12}^{2} & x & 0 \\
1 & l_{2 A}^{2} & 0 & 0 & 0 & y \\
1 & l_{3 A}^{2} & x & l_{32}^{2} & 0 & 0 \\
1 & l_{4 A}^{2} & 0 & 0 & 0 & 0
\end{array}\right|+\left|\begin{array}{cccccc}
0 & 1 & 0 & 0 & 0 & 0 \\
1 & 0 & 0 & 0 & 0 & 0 \\
1 & l_{1 A}^{2} & 0 & 0 & x & 0 \\
1 & l_{2 A}^{2} & 0 & 0 & 0 & y \\
1 & l_{3 A}^{2} & x & 0 & 0 & 0 \\
1 & l_{4 A}^{2} & 0 & y & 0 & 0
\end{array}\right| \\
& +\left|\begin{array}{cccccc}
0 & 1 & 0 & 1 & 0 & 1 \\
1 & 0 & 0 & l_{A 2}^{2} & 0 & l_{A 4}^{2} \\
1 & l_{1 A}^{2} & 0 & l_{12}^{2} & x & l_{14}^{2} \\
1 & l_{2 A}^{2} & 0 & 0 & 0 & 0 \\
1 & l_{3 A}^{2} & x & l_{32}^{2} & 0 & l_{34}^{2} \\
1 & l_{4 A}^{2} & 0 & 0 & 0 & 0
\end{array}\right|+\left|\begin{array}{cccccc}
0 & 1 & 0 & 0 & 0 & 1 \\
1 & 0 & 0 & 0 & 0 & l_{A 4}^{2} \\
1 & l_{1 A}^{2} & 0 & 0 & x & l_{14}^{2} \\
1 & l_{2 A}^{2} & 0 & 0 & 0 & 0 \\
1 & l_{3 A}^{2} & x & 0 & 0 & l_{34}^{2} \\
1 & l_{4 A}^{2} & 0 & y & 0 & 0
\end{array}\right| \\
& +\left|\begin{array}{cccccc}
0 & 1 & 1 & 1 & 1 & 1 \\
1 & 0 & l_{A 1}^{2} & l_{A 2}^{2} & l_{A 3}^{2} & l_{A 4}^{2} \\
1 & l_{1 A}^{2} & 0 & l_{12}^{2} & 0 & l_{14}^{2} \\
1 & l_{2 A}^{2} & l_{12}^{2} & 0 & l_{23}^{2} & 0 \\
1 & l_{3 A}^{2} & 0 & l_{32}^{2} & 0 & l_{34}^{2} \\
1 & l_{4 A}^{2} & l_{41}^{2} & 0 & l_{34}^{2} & 0
\end{array}\right|+\left|\begin{array}{cccccc}
0 & 1 & 1 & 1 & 1 & 0 \\
1 & 0 & l_{A 1}^{2} & l_{A 2}^{2} & l_{A 3}^{2} & 0 \\
1 & l_{1 A}^{2} & 0 & l_{12}^{2} & 0 & 0 \\
1 & l_{2 A}^{2} & l_{12}^{2} & 0 & l_{23}^{2} & 0 \\
1 & l_{3 A}^{2} & 0 & l_{32}^{2} & 0 & 0 \\
1 & l_{4 A}^{2} & l_{41}^{2} & 0 & l_{34}^{2} & 0
\end{array}\right| \\
& +\left|\begin{array}{cccccc}
0 & 1 & 1 & 0 & 1 & 1 \\
1 & 0 & l_{A 1}^{2} & 0 & l_{A 3}^{2} & l_{A 4}^{2} \\
1 & l_{1 A}^{2} & 0 & 0 & 0 & l_{14}^{2} \\
1 & l_{2 A}^{2} & l_{12}^{2} & 0 & l_{23}^{2} & 0 \\
1 & l_{3 A}^{2} & 0 & 0 & 0 & l_{34}^{2} \\
1 & l_{4 A}^{2} & l_{41}^{2} & y & l_{34}^{2} & 0
\end{array}\right|+\left|\begin{array}{cccccc}
0 & 1 & 1 & 0 & 1 & 0 \\
1 & 0 & l_{A 1}^{2} & 0 & l_{A 3}^{2} & 0 \\
1 & l_{1 A}^{2} & 0 & 0 & 0 x & 0 \\
1 & l_{2 A}^{2} & l_{12}^{2} & 0 & l_{23}^{2} & y \\
1 & l_{3 A}^{2} & 0 & 0 & 0 & 0 \\
1 & l_{4 A}^{2} & l_{41}^{2} & y & l_{34}^{2} & 0
\end{array}\right| \\
& +\left|\begin{array}{cccccc}
0 & 1 & 0 & 1 & 1 & 1 \\
1 & 0 & 0 & l_{A 2}^{2} & l_{A 3}^{2} & l_{A 4}^{2} \\
1 & l_{1 A}^{2} & 0 & l_{12}^{2} & 0 & l_{14}^{2} \\
1 & l_{2 A}^{2} & 0 & 0 & l_{23}^{2} & 0 \\
1 & l_{3 A}^{2} & x & l_{32}^{2} & 0 & l_{34}^{2} \\
1 & l_{4 A}^{2} & 0 & 0 & l_{34}^{2} & 0
\end{array}\right|+\left|\begin{array}{cccccc}
0 & 1 & 0 & 1 & 1 & 0 \\
1 & 0 & 0 & l_{A 2}^{2} & l_{A 3}^{2} & 0 \\
1 & l_{1 A}^{2} & 0 & l_{12}^{2} & 0 & 0 \\
1 & l_{2 A}^{2} & 0 & 0 & l_{23}^{2} & y \\
1 & l_{3 A}^{2} & x & l_{32}^{2} & 0 & 0 \\
1 & l_{4 A}^{2} & 0 & 0 & l_{34}^{2} & 0
\end{array}\right| \\
& +\left|\begin{array}{cccccc}
0 & 1 & 0 & 0 & 1 & 1 \\
1 & 0 & 0 & 0 & l_{A 3}^{2} & l_{A 4}^{2} \\
1 & l_{1 A}^{2} & 0 & 0 & x & l_{14}^{2} \\
1 & l_{2 A}^{2} & 0 & 0 & l_{23}^{2} & 0 \\
1 & l_{3 A}^{2} & x & 0 & 0 & l_{34}^{2} \\
1 & l_{4 A}^{2} & 0 & y & l_{34}^{2} & 0
\end{array}\right|+\left|\begin{array}{cccccc}
0 & 1 & 0 & 0 & 1 & 0 \\
1 & 0 & 0 & 0 & l_{A 3}^{2} & 0 \\
1 & l_{1 A}^{2} & 0 & 0 & 0 & 0 \\
1 & l_{2 A}^{2} & 0 & 0 & l_{23}^{2} & y \\
1 & l_{3 A}^{2} & x & 0 & 0 & 0 \\
1 & l_{4 A}^{2} & 0 & y & l_{34}^{2} & 0
\end{array}\right| .
\end{aligned}
$$

Denote each determinant in the above sum by $\Delta_{i}, 1 \leq i \leq 16$, where the number $i$ corresponds to the position of $\Delta_{i}$ as the summand in this sum. The direct calculation of each $\Delta_{i}$ gives

$$
\Delta_{1}=\Delta_{13}=x\left|\begin{array}{ccccc}
0 & 1 & 1 & 1 & 1 \\
1 & 0 & l_{A 1}^{2} & l_{A 2}^{2} & l_{A 4}^{2} \\
1 & l_{2 A}^{2} & l_{21}^{2} & 0 & 0 \\
1 & l_{3 A}^{2} & 0 & l_{32}^{2} & l_{34}^{2} \\
1 & l_{4 A}^{2} & l_{41}^{2} & 0 & 0
\end{array}\right|
$$




$$
\begin{aligned}
& \Delta_{2}+\Delta_{3}=x y\left(\begin{array}{cccc}
0 & 1 & 1 & 1 \\
1 & 0 & l_{A 1}^{2} & l_{A 2}^{2} \\
1 & l_{3 A}^{2} & 0 & l_{32}^{2} \\
1 & l_{4 A}^{2} & l_{41}^{2} & 0
\end{array}|-| \begin{array}{cccc}
0 & 1 & 1 & 1 \\
1 & 0 & l_{A 1}^{2} & l_{A 4}^{2} \\
1 & l_{2 A}^{2} & l_{21}^{2} & 0 \\
1 & l_{3 A}^{2} & 0 & l_{34}^{2}
\end{array} \mid\right), \\
& \Delta_{4}=\Delta_{16}=x y^{2}\left(l_{1 A}^{2}+l_{3 A}^{2}\right), \\
& \Delta_{5}=\Delta_{8}=y x^{2}\left(l_{2 A}^{2}+l_{4 A}^{2}\right), \\
& \Delta_{6}=-x^{2} y^{2}, \quad \Delta_{7}=-x^{2}\left(l_{2 A}^{2}-l_{4 A}^{2}\right)^{2}, \quad \Delta_{12}=-y^{2}\left(l_{1 A}^{2}-l_{3 A}^{2}\right)^{2}, \\
& \Delta_{10}=\Delta_{11}=y\left|\begin{array}{ccccc}
0 & 1 & 1 & 1 & 1 \\
1 & 0 & l_{A 1}^{2} & l_{A 2}^{2} & l_{A 3}^{2} \\
1 & l_{2 A}^{2} & 0 & l_{12}^{2} & 0 \\
1 & l_{3 A}^{2} & 0 & l_{32}^{2} & 0 \\
1 & l_{4 A}^{2} & l_{41}^{2} & 0 & l_{34}^{2}
\end{array}\right| \\
& \Delta_{14}+\Delta_{15}=x y\left(\left|\begin{array}{cccc}
0 & 1 & 1 & 1 \\
1 & 0 & l_{A 3}^{2} & l_{A 4}^{2} \\
1 & l_{1 A}^{2} & 0 & l_{14}^{2} \\
1 & l_{2 A}^{2} & l_{23}^{2} & 0
\end{array}\right|-\left|\begin{array}{cccc}
0 & 1 & 1 & 1 \\
1 & 0 & l_{A 2}^{2} & l_{A 3}^{2} \\
1 & l_{1 A}^{2} & l_{12}^{2} & 0 \\
1 & l_{4 A}^{2} & 0 & l_{34}^{2}
\end{array}\right|\right)
\end{aligned}
$$

and we leave $\Delta_{9}$ without any change so far:

$$
\Delta_{9}=\left|\begin{array}{cccccc}
0 & 1 & 1 & 1 & 1 & 1 \\
1 & 0 & l_{A 1}^{2} & l_{A 2}^{2} & l_{A 3}^{2} & l_{A 4}^{2} \\
1 & l_{1 A}^{2} & 0 & l_{12}^{2} & 0 & l_{14}^{2} \\
1 & l_{2 A}^{2} & l_{12}^{2} & 0 & l_{23}^{2} & 0 \\
1 & l_{3 A}^{2} & 0 & l_{32}^{2} & 0 & l_{34}^{2} \\
1 & l_{4 A}^{2} & l_{41}^{2} & 0 & l_{34}^{2} & 0
\end{array}\right| .
$$

Now we introduce new notations: $l_{12}^{2}=p, l_{14}^{2}=q$. Calculating in a similar fashion, we obtain:

$$
\begin{aligned}
\Delta_{1}+\Delta_{13}=2 x & \left(\begin{array}{ccccc}
0 & 1 & 1 & 1 & 1 \\
1 & 0 & l_{2 A}^{2} & l_{3 A}^{2} & l_{4 A}^{2} \\
1 & l_{1 A}^{2} & 0 & 0 & 0 \\
1 & l_{2 A}^{2} & 0 & l_{23}^{2} & 0 \\
1 & l_{4 A}^{2} & 0 & l_{34}^{2} & 0
\end{array}|+p| \begin{array}{cccc}
0 & 1 & 1 & 1 \\
1 & 0 & l_{3 A}^{2} & l_{4 A}^{2} \\
1 & l_{2 A}^{2} & l_{23}^{2} & 0 \\
1 & l_{4 A}^{2} & l_{34}^{2} & 0
\end{array} \mid\right. \\
& \left.+q\left|\begin{array}{cccc}
0 & 1 & 1 & 1 \\
1 & 0 & l_{2 A}^{2} & l_{3 A}^{2} \\
1 & l_{2 A}^{2} & 0 & l_{23}^{2} \\
1 & l_{4 A}^{2} & 0 & l_{34}^{2}
\end{array}\right|\right),
\end{aligned}
$$




$$
\begin{aligned}
& \Delta_{10}+\Delta_{11}=2 y\left(2 p q l_{3 A}^{2}+\left|\begin{array}{ccccc}
0 & 1 & 1 & 1 & 1 \\
1 & 0 & l_{1 A}^{2} & l_{2 A}^{2} & l_{3 A}^{2} \\
1 & l_{1 A}^{2} & 0 & 0 & 0 \\
1 & l_{3 A}^{2} & 0 & l_{23}^{2} & 0 \\
1 & l_{4 A}^{2} & 0 & 0 & l_{34}^{2}
\end{array}\right|-p\left|\begin{array}{cccc}
0 & 1 & 1 & 1 \\
1 & 0 & l_{A 1}^{2} & l_{A 3}^{2} \\
1 & l_{3 A}^{2} & 0 & 0 \\
1 & l_{4 A}^{2} & 0 & l_{34}^{2}
\end{array}\right|\right. \\
& +q\left(\begin{array}{cccc}
0 & 1 & 1 & 1 \\
1 & 0 & l_{A 2}^{2} & l_{A 3}^{2} \\
1 & l_{1 A}^{2} & 0 & 0 \\
1 & l_{3 A}^{2} & l_{23}^{2} & 0
\end{array} \mid\right) \\
& \Delta_{2}+\Delta_{3}+\Delta_{14}+\Delta_{15}=2 x y\left(\left|\begin{array}{cccc}
0 & 1 & 1 & 1 \\
1 & 0 & l_{1 A}^{2} & l_{2 A}^{2} \\
1 & l_{3 A}^{2} & 0 & l_{23}^{2} \\
1 & l_{4 A}^{2} & 0 & 0
\end{array}\right|-\left|\begin{array}{cccc}
0 & 1 & 1 & 1 \\
1 & 0 & l_{2 A}^{2} & l_{3 A}^{2} \\
1 & l_{1 A}^{2} & 0 & 0 \\
1 & l_{4 A}^{2} & 0 & l_{34}^{2}
\end{array}\right|\right. \\
& \left.-q\left|\begin{array}{ccc}
0 & 1 & 1 \\
1 & 0 & l_{2 A}^{2} \\
1 & l_{3 A}^{2} & l_{13}^{2}
\end{array}\right|-p\left|\begin{array}{ccc}
0 & 1 & 1 \\
1 & 0 & l_{3 A}^{2} \\
1 & l_{4 A}^{2} & l_{34}^{2}
\end{array}\right|\right) \text {, }
\end{aligned}
$$$$
\Delta_{9}=\Delta_{17}-p\left(\Delta_{18}+\Delta_{19}\right)-q\left(\Delta_{20}+\Delta_{21}\right)=-2 p q \Delta_{22}+p^{2} \Delta_{23}+q^{2} \Delta_{24}, \text { where }
$$

$$
\begin{aligned}
& \Delta_{17}=\left|\begin{array}{cccccc}
0 & 1 & 1 & 1 & 1 & 1 \\
1 & 0 & l_{1 A}^{2} & l_{2 A}^{2} & l_{3 A}^{2} & l_{4 A}^{2} \\
1 & l_{1 A}^{2} & 0 & 0 & 0 & 0 \\
1 & l_{2 A}^{2} & 0 & 0 & l_{23}^{2} & 0 \\
1 & l_{3 A}^{2} & 0 & l_{23}^{2} & 0 & l_{34}^{2} \\
1 & l_{4 A}^{2} & 0 & 0 & l_{34}^{2} & 0
\end{array}\right|, \quad \Delta_{22}=\left|\begin{array}{cccc}
0 & 1 & 1 & 1 \\
1 & 0 & l_{2 A}^{2} & l_{3 A}^{2} \\
1 & l_{3 A}^{2} & l_{23}^{2} & 0 \\
1 & l_{4 A}^{2} & 0 & l_{34}^{2}
\end{array}\right| \\
& \Delta_{18}=\left|\begin{array}{ccccc}
0 & 1 & 1 & 1 & 1 \\
1 & 0 & l_{1 A}^{2} & l_{3 A}^{2} & l_{4 A}^{2} \\
1 & l_{2 A}^{2} & 0 & l_{23}^{2} & 0 \\
1 & l_{3 A}^{2} & 0 & 0 & l_{34}^{2} \\
1 & l_{4 A}^{2} & 0 & l_{34}^{2} & 0
\end{array}\right|, \quad \quad \Delta_{19}=\left|\begin{array}{ccccc}
0 & 1 & 1 & 1 & 1 \\
1 & 0 & l_{2 A}^{2} & l_{3 A}^{2} & l_{4 A}^{2} \\
1 & l_{1 A}^{2} & 0 & 0 & 0 \\
1 & l_{3 A}^{2} & l_{23}^{2} & 0 & l_{34}^{2} \\
1 & l_{4 A}^{2} & 0 & l_{34}^{2} & 0
\end{array}\right|, \\
& \Delta_{20}=\left|\begin{array}{ccccc}
0 & 1 & 1 & 1 & 1 \\
1 & 0 & l_{1 A}^{2} & l_{2 A}^{2} & l_{3 A}^{2} \\
1 & l_{2 A}^{2} & 0 & 0 & l_{23}^{2} \\
1 & l_{3 A}^{2} & 0 & l_{23}^{2} & 0 \\
1 & l_{4 A}^{2} & 0 & 0 & l_{34}^{2}
\end{array}\right|, \quad \Delta_{21}=\left|\begin{array}{ccccc}
0 & 1 & 1 & 1 & 1 \\
1 & 0 & l_{2 A}^{2} & l_{3 A}^{2} & l_{4 A}^{2} \\
1 & l_{1 A}^{2} & 0 & 0 & 0 \\
1 & l_{2 A}^{2} & 0 & l_{23}^{2} & 0 \\
1 & l_{3 A}^{2} & l_{23}^{2} & 0 & l_{34}^{2}
\end{array}\right| \\
& \Delta_{23}=-\left|\begin{array}{cccc}
0 & 1 & 1 & 1 \\
1 & 0 & l_{3 A}^{2} & l_{4 A}^{2} \\
1 & l_{3 A}^{2} & 0 & l_{34}^{2} \\
1 & l_{4 A}^{2} & l_{34}^{2} & 0
\end{array}\right|, \quad \Delta_{24}=-\left|\begin{array}{cccc}
0 & 1 & 1 & 1 \\
1 & 0 & l_{3 A}^{2} & l_{4 A}^{2} \\
1 & l_{2 A}^{2} & l_{23}^{2} & 0 \\
1 & l_{3 A}^{2} & 0 & l_{34}^{2}
\end{array}\right| .
\end{aligned}
$$

Note that the coefficient $\Delta_{23}$ at $p^{2}$ is equal to $16 \mathrm{vol}^{2}$ (triangle $\left\langle p_{3}, p_{4}, A\right\rangle$ ) and that at $q^{2}$ is $16 \mathrm{vol}^{2}$ (triangle $\left\langle p_{2}, p_{3}, A\right\rangle$ ). 
Later we heavily use the Cayley-Menger equation whenever the points $A, p_{1}, p_{2}, p_{3}$, $p_{4}$ are the vertices of a polyhedron $P$ in a general configuration (this means that the coordinates of the vertices are algebraically independent; in particular, no three vertices lie on the same line). For this reason we have to present this equation in a short and, on the other hand, sufficiently informative form. Denote by $l_{A}$ the set $\left(l_{1 A}^{2}, l_{2 A}^{2}, l_{3 A}^{2}, l_{4 A}^{2}, l_{23}^{2}, l_{34}^{2}\right)$. The Cayley-Menger determinant $\Delta$ can be regarded as a polynomial over the variables $x, y, p, q$ with coefficients depending on $l_{A}$.

When no three of five points $A, p_{1}, p_{2}, p_{3}, p_{4}$ lie on the same line, some coefficients are certainly distinct from zero, for example, $\Delta_{23}$ and $\Delta_{24}$ at $p^{2}$ and $q^{2}$. We denote all of them by subscripted $a$. The coefficients that can be equal to zero, for some specific values of $\left(l_{A}\right)$, are denoted by $b$ with a subscript ${ }^{1}$; for example, the coefficients at $x$ and $y$ are so. Now the Cayley-Menger equation $-\Delta=0$ can be presented in the following form:

$$
\begin{aligned}
-\Delta= & -\Delta_{6}-\left(\Delta_{5}+\Delta_{8}\right)-\left(\Delta_{7}+\Delta_{12}\right)-\left(\Delta_{4}+\Delta_{16}\right) \\
& -\left(\Delta_{10}+\Delta_{11}\right)-\left(\Delta_{1}+\Delta_{13}\right)-\left(\Delta_{2}+\Delta_{3}+\Delta_{14}+\Delta_{15}\right)-\Delta_{9} \\
= & x^{2}\left[y^{2}+a_{1}\left(l_{A}\right) y+b_{1}\left(l_{A}\right)\right] \\
& +x\left[a_{2}\left(l_{A}\right) y^{2}+b_{2}\left(l_{A}\right) p y+b_{3}\left(l_{A}\right) q y+b_{4}\left(l_{A}\right) p+b_{5}\left(l_{A}\right) q+b_{6}\left(l_{A}\right) y+b_{7}\left(l_{A}\right)\right] \\
& +\left[a_{3}\left(l_{A}\right) p^{2}+a_{4}\left(l_{A}\right) q^{2}+b_{8}\left(l_{A}\right) y^{2}+a_{5}\left(l_{A}\right) p q y+b_{10}\left(l_{A}\right) p q+b_{11}\left(l_{A}\right) p y\right. \\
& \left.\quad+b_{12}\left(l_{A}\right) q y+b_{13}\left(l_{A}\right) p+b_{14}\left(l_{A}\right) q+b_{15}\left(l_{A}\right) y+b_{16}\left(l_{A}\right)\right] \\
= & 0
\end{aligned}
$$

We will also need some interpretation of the Caley-Menger equation as a polynomial equation for one of the variables $p$ or $q$. Therefore it is useful to have this presentation, say, for $p$ :

$$
\begin{aligned}
& a p^{2}+(a q y+b x y+b q+b x+b y+b) p \\
&+\left(x^{2} y^{2}+a x y^{2}+a y x^{2}+a q^{2}+b x^{2}+b y^{2}+b q x y\right. \\
&+b q x+b q y+b x y+b q+b x+b y+b)=0 .
\end{aligned}
$$

Here we omit the subscripts for the coefficients because we only pay attention to the distribution of the degrees of $x, y, q$ between the coefficients for $p^{2}, p$, and $p^{0}$. (For example, note that there is no monomial $x p q$.) The equation for $q$ has the same form.

Remark. It is essential to notice that the coefficient $\left[y^{2}+a\left(l_{A}\right) y+b\left(l_{A}\right)\right]$ at $x^{2}$ is equal to $l_{34}^{4}+l_{2 A}^{4}+l_{4 A}^{4}+2 l_{2 A}^{2} l_{4 A}^{2}-2 l_{34}^{2} l_{4 A}^{2}-2 l_{2 A}^{2} l_{34}^{2}=-16 \mathrm{vol}^{2}\left(\right.$ triangle $\left.\left\langle p_{2}, p_{4}, A\right\rangle\right)$ so it is nonzero for any general configuration of points.

\section{The Main Lemma}

First we fix our terminology. We say that a polyhedron $P$ has the property $Q$ if for $P$ and for all polyhedra from $\tilde{P}$ the above theorem is valid.

\footnotetext{
${ }^{1}$ In fact, for polyhedron $P$ in a general position none of Cayley-Menger coefficients is equal to zero; thus we distinguish them by the equality or the nonequality to zero under the simplest condition that there be no three points lying on the same line in $P$.
} 
We say that a 3-cycle (a cycle composed by three edges) $L$ is empty if it is not the boundary of a face of the polyhedron $P$. For example, in the polyhedron of Fig. 2 the 3-cycle $\left\langle A p_{2} p_{3}\right\rangle$ is empty.

We say that a simplicial complex $K$ is of class $K_{0}$ if $K$ has at least one vertex which is not incident to any empty 3-cycle. Note that if $K$ has a vertex of degree 3, then $K$ is of class $K_{0}$.

Now we can formulate our next lemma.

Lemma 1. Suppose that all the polyhedra with $n$ vertices and given genus $g \geq 0$ have the property $Q$. Let $P$ be a polyhedron with $n+1$ vertices which has the combinatorial structure of class $K_{0}$ with the same genus $g$. Then $P$ has the property $Q$ too.

\section{Proof of the Lemma}

Suppose first that a polyhedron $P$ with $n+1$ vertices and of combinatorial type $K_{0}$ is in the general position, i.e., the coordinates of its vertices are algebraically independent. We proceed by induction on the degree $m$ of a vertex which is not incident to any empty 3-cycle. If $P$ has a vertex $A$ of degree $m=3$, then the lemma is evident. Before making the induction step "from $m$ to $m+1$ " with an arbitrary $m$, we dwell on the step "from 3 to 4," which will help us demonstrate our idea for the general case. So, let $A$ be a vertex of degree 4 which is not incident to any empty 3-cycle and let St $A$ denote the star of the vertex $A$. Number the vertices of the boundary of St $A$ in a cyclic order, say, $p_{0}, p_{1}, p_{2}, p_{3}$ starting from an arbitrary vertex. On the base of $P$ we construct two polyhedra $P_{1}$ and $P_{2}$, both having a vertex of degree 3 . Namely, for the construction of $P_{i}$ we remove the edge $\left\langle A p_{i}\right\rangle, i=1,2$, with the two incident faces $\left\langle p_{i-1} A p_{i}\right\rangle,\left\langle p_{i} A p_{i+1}\right\rangle$, and then replace them by new faces $\left\langle p_{i-1} p_{i} p_{i+1}\right\rangle$ and $\left\langle p_{i-1} A p_{i+1}\right\rangle$; see Fig. 6. These operations are possible because the diagonals $\left\langle p_{0} p_{2}\right\rangle$ and $\left\langle p_{1} p_{3}\right\rangle$ are not edges of $P$ and therefore in the new polyhedra $P_{1}$ and $P_{2}$ both are incident exactly to two faces.

The vertex $A$ is of degree 3 in $P_{1}$ and $P_{2}$. Denote the lengths of the diagonals $\left\langle p_{0} p_{2}\right\rangle$
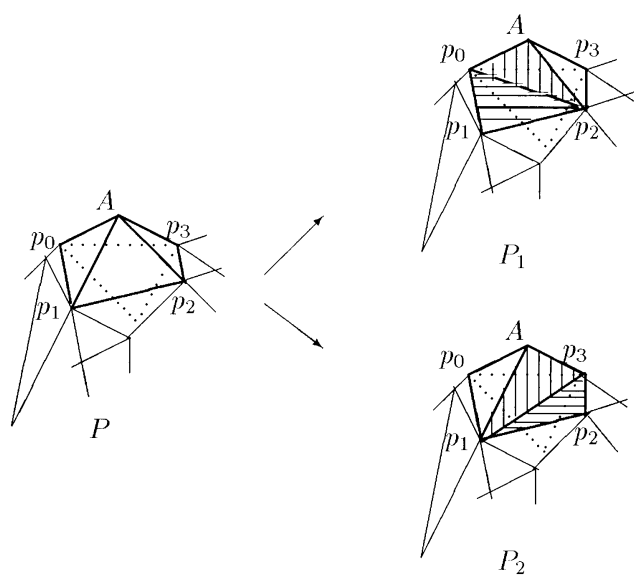

Fig. 6 
and $\left\langle p_{1} p_{3}\right\rangle$ by $d_{1}$ and $d_{2}$, respectively; the subscript indicates the number of the point "between" the ends of the corresponding diagonal. By the induction base, the volumes $V_{1}=\operatorname{vol}\left(P_{1}\right)$ and $V_{2}=\operatorname{vol}\left(P_{2}\right)$ are roots of some polynomial equations of the form

$$
Q_{i}=V_{i}^{2 N_{i}}+a_{1}^{(i)}\left(l, d_{i}^{2}\right) V_{i}^{2 N_{i}-2}+\cdots=0, \quad i=1,2 .
$$

Let $V_{0 i}$ be the volume of the tetrahedron $\left\langle A p_{i-1} p_{i} p_{i+1}\right\rangle$ completing $P_{i}$ up to $P$. Then for the volume $V(P)$ we have

$$
V_{i}=V-\varepsilon V_{0 i}, \quad \varepsilon= \pm 1, \quad i=1,2 .
$$

Substituting the value $V_{i}$ from $\left(9_{i}\right)$ to $\left(8_{i}\right)$, and using the equalities

$$
V_{i}^{2 m}=\sum_{k=1}^{m-1} C_{2 m}^{2 k} V^{2 k} V_{0 i}^{2(n-k)}-\varepsilon V V_{0 i} \sum_{k=0}^{m-1} C_{2 m}^{2 k+1} V^{2 k} V_{0 i}^{2(m-k-1)}, \quad i=1,2, \ldots,
$$

we obtain (after routine algebraic manipulations) equations of the form

$$
V^{4 N_{i}}+A_{1}^{(i)}\left(l, d_{i}^{2}, V_{0 i}^{2}\right) V^{4 N_{i}-2}+\cdots+A_{2 N_{i}}^{(i)}\left(l, d_{i}^{2}, V_{0 i}^{2}\right) V^{0}=0,
$$

already without $\varepsilon$. It means that these equations do not depend on a particular position of St $A$ relatively to the rest part of $P$. Now, in $\left(10_{i}\right)$ substitute the value of $V_{0 i}^{2}$ accordingly (1) as functions of $\left(l, d_{i}^{2}\right)$ and get

$$
V^{4 N_{i}}+B_{1}^{(i)}\left(l, d_{i}^{2}\right) V^{4 N_{i}-2}+\cdots+B_{2 N_{i}}^{(i)}\left(l, d_{i}^{2}\right) V^{0}=0, \quad i=1,2 .
$$

We can consider $\left(11_{i}\right)$ also as polynomial equations in $d_{i}^{2}$. For this, present $\left(11_{i}\right)$ as follows:

$$
C_{0}^{(i)}\left(l, V^{2}\right) d_{i}^{2 K i}+\cdots+C_{K_{i}}^{(i)}\left(l, V^{2}\right)=0, \quad i=1,2,
$$

where $2 K_{i} \leq 12 N_{i}$ is the maximum of the $d_{i}$ 's degrees in the polynomial equation $\left(11_{i}\right)$ when $(l)=\left(l_{1}^{2}, \ldots, l_{e}^{2}\right)$ are regarded as independent variables. If for $i=1$ or 2 one has $K_{i}=0$, then the corresponding equation $\left(12_{i}\right)$ takes the form $C_{K_{i}}^{(i)}\left(l, V^{2}\right)=$ $V^{4 N_{i}}+\cdots=0$, so that for $V$ we have the required equation $Q(V)=V^{2 N}+\cdots=0$ with $N=2 N_{i}$. Let $K_{i}>0, i=1,2$. Substitute in $C_{j}^{(i)}\left(l, V^{2}\right), 0 \leq j<K_{i}$, the value of $l=\left(l_{1}^{2}, \ldots, l_{e}^{2}\right)$ and $V$ as polynomial functions of $\left(x_{1}, \ldots, z_{n}\right)$. If all functions obtained by this procedure are identical to zero, again we come to a desired equation $Q(V)=C_{K_{i}}^{(i)}\left(l, V^{2}\right)=V^{4 N_{i}}+\cdots=0$. If at least one of these functions is not identical to zero, then for $P$ the coefficient at the corresponding degree of $d_{i}$ is not equal to zero because $P$ is in a general position. For brevity, we suppose that the first such coefficient is $C_{0}^{(i)}\left(l, V^{2}\right)$, so we have equations of the form $\left(12_{i}\right)$ with $C_{0}^{(i)}\left(l, V^{2}\right) \neq 0, i=1,2$.

Now we recall that ten distances between five points $A, p_{0}, p_{1}, p_{2}, p_{3}$ satisfy the Cayley-Menger equation (6). In our case $x=d_{1}^{2}, y=d_{2}^{2}$ and other distances, in particular $p$ and $q$, are the lengths of the edges of $P$. Therefore we can denote them all by $(l)$. Then the corresponding Cayley-Menger equation can be rewritten in the following form:

$$
\bar{d}_{1}^{2}\left(\bar{d}_{2}^{2}+a \bar{d}_{2}+b\right)+\bar{d}_{1}\left(a \bar{d}_{2}^{2}+b \bar{d}_{2}+b\right)+\left(b \bar{d}_{2}^{2}+b \bar{d}_{2}+b\right)=0
$$


where $\bar{d}_{1}=d_{1}^{2}, \bar{d}_{2}=d_{2}^{2}$ (we do not write the variables $p$ and $q$ because in the case in question they are known to be the lengths of the edges $\left\langle p_{0} p_{1}\right\rangle$ and $\left.\left\langle p_{0} p_{3}\right\rangle\right)$. Furthermore, we omit the subscripts of the coefficients $a$ and $b$. Since the leading coefficients in $\left(12_{1}\right)$ and (13) are different from zero, we can eliminate $\bar{d}_{1}$ by using the resultant of $\left(12_{1}\right)$ and (13). This procedure leads to the following equation:

$$
\left|\begin{array}{cccccc}
\left(\bar{d}_{2}^{2}+\cdots\right) & \left(a \bar{d}_{2}^{2}+\cdots\right) & \left(b \bar{d}_{2}^{2}+\cdots\right) & * & \cdots & 0 \\
0 & \left(\bar{d}_{2}^{2}+\cdots\right) & \cdots & \cdots & \cdots & 0 \\
\vdots & \ddots & \vdots & \vdots & \vdots & \vdots \\
0 & \cdots & \left(\bar{d}_{2}^{2}+\cdots\right) & \cdots & \cdots & * \\
C_{0}^{(1)}\left(l, V^{2}\right) & C_{1}^{(1)}\left(l, V^{2}\right) & \cdots & \left(V^{4 N_{1}}+\cdots\right) & \cdots & 0 \\
0 & \cdots & C_{0}^{(1)}\left(l, V^{2}\right) & \cdots & \cdots & \left(V^{4 N_{1}}+\cdots\right),
\end{array}\right|=0
$$

where by " $*$ " we denote the coefficient at $\bar{d}_{1}^{0}$ in (13). We recall that in the coefficients $C_{j}^{(1)}$ the degree of $V$ is less than $4 N_{1}$. Therefore, calculating this determinant of size $\left(K_{1}+2\right) \times\left(K_{1}+2\right)$, we obtain the following equation:

$\bar{d}_{2}^{2 K_{1}}\left(V^{8 N_{1}}+\cdots\right)+\bar{d}_{2}^{2 K_{1}-1}\left(a V^{8 N_{1}}+\cdots\right)+\bar{d}_{2}^{2 K_{1}-2}\left(b V^{8 N_{1}}+\cdots\right)+\cdots+\bar{d}_{2}^{0}\left(b V^{8 N_{1}}+\cdots\right)=0$.

Now we can eliminate $\bar{d}_{2}$ from (14), and rewrite $\left(12_{2}\right)$ for $\bar{d}_{2}=d_{2}^{2}$. It is easy to see that the resultant equation is as follows:

$$
\left|\begin{array}{cccccc}
V^{8 N_{1}}+\cdots & a V^{8 N_{1}}+\cdots & \cdots & * & \cdots & 0 \\
0 & V^{8 N_{1}}+\cdots & \cdots & \cdots & \cdots & \cdots \\
\vdots & \ddots & \vdots & \vdots & \cdots & \cdots \\
0 & \cdots & V^{8 N_{1}}+\cdots & \cdots & \cdots & * \\
C_{0}^{(2)}\left(l, V^{2}\right) & C_{1}^{(2)}\left(l, V^{2}\right) & \cdots & V^{4 N_{2}}+\cdots & \cdots & 0 \\
\vdots & \ddots & \vdots & \ddots & \vdots & \\
0 & \cdots & C_{0}^{(2)}\left(l, V^{2}\right) & \cdots & \cdots & V^{4 N_{2}}+\cdots
\end{array}\right|=0 .
$$

It follows that the greatest degree of $V$ is $2 N=8 K_{2} N_{1}+8 K_{1} N_{2}$ (because the degree of $V$ in the coefficients $C_{i}^{(2)}$ is less than $4 N_{2}$ ) and the leading coefficient is 1 . Therefore, we have come to the desired equation $Q(V)=V^{2 N}+\cdots=0$, where the degree $2 N$ of $Q(V)$ satisfies $2 N \leq 96 N_{1} N_{2}$. Thus, if the degree of the vertex $A$ is 4 , the induction step has been performed.

Suppose now that the lemma has been proved for vertices of degree less than or equal to $m$, and consider the case where vertex $A$ has degree $m+1$. Note that for the case of a vertex of degree 4 the elimination process used in our considerations turned out to be possible due to the availability of relation (13) between the diagonals and edges of St $(A)$. So, proceeding to the study of the general case, we should try to find also a relation between the edges and diagonals of St $(A)$ when vertex $A$ is of degree $m+1$. However, first we obtain analogs of $\left(11_{i}\right)$ and $\left(12_{i}\right)$ starting with the construction of new polyhedra $P_{i}, 1 \leq i \leq m-1$, in which vertex $A$ has degree $m$. For this, we denote the vertices on the boundary of ( $\mathrm{St} A$ ) (in a cyclic order) by $p_{0}, p_{1}, \ldots, p_{m}$ starting from some vertex and replacing the two faces incident to the edge $A p_{i}$ by new faces, 
$\left\langle p_{i-1} A p_{i+1}\right\rangle$ and $\left\langle p_{i-1} p_{i} p_{i+1}\right\rangle$, incident to the diagonal $\left\langle p_{i-1} p_{i+1}\right\rangle$. Denote by $d_{i}$ the length of this diagonal. Then, by the induction hypothesis, $V_{i}=\operatorname{vol}\left(P_{i}\right)$ is a root of some polynomial equation of the following form:

$$
V_{i}^{2 N_{i}}+a_{1}^{(i)}\left(l, d_{i}\right) V_{i}^{2 N_{i}-2}+\cdots=0, \quad 0 \leq i \leq m .
$$

Furthermore, by the same argument as above for a vertex of degree 4, we come to the conclusion that $V(P)$ and $d_{i}$ satisfy the following equation:

$$
V^{4 N_{i}}+B_{1}^{(i)}\left(l, d_{i}^{2}\right) V^{4 N_{i}-2}+\cdots+B_{2 N_{i}}^{(i)}\left(l, d_{i}^{2}\right) V^{0}=0, \quad 0 \leq i \leq m .
$$

(These equations are written for all vertices of $\partial(\operatorname{St} A)$, but for the construction of $P_{i}$ we use only those corresponding to $i=1, \ldots, m-1$.) Equation $\left(16_{i}\right)$ can be presented also as a polynomial equation for $d_{i}$ as follows:

$$
C_{0}^{(i)}\left(l, V^{2}\right) d_{i}^{2 K_{i}}+\cdots+C_{K_{i}}^{(i)}\left(l, V^{2}\right)=0
$$

where $C_{K_{i}}^{(i)}\left(l, V^{2}\right)=V^{4 N_{i}}+\cdots$.

If at least one of equations $\left(17_{i}\right)$ does not depend on $d_{i}$ for some $i$ when $l_{1}^{2}, \ldots, l_{e}^{2}$ are regarded as independent variables, then we already have the required polynomial equation for $V(P): Q(V)=C_{K_{i}}^{(i)}\left(l, V^{2}\right)=V^{4 N_{i}}+\cdots=0$. Consider now another possible case when the coefficients at $d_{i}$ are identical to zero if $l$ and $V$ are replaced by their expressions as functions of vertex coordinates $\left(x_{1}, \ldots, z_{n}\right)$. In this case we also have the required equation for $V: C_{K_{i}}^{(i)}\left(l, V^{2}\right)=0$. So we can assume that all $K_{i}>0$ and (after taking into account the fact that polyhedron $P$ is in the general position) that all the coefficients $C_{0}^{(i)}\left(l, V^{2}\right) \neq 0$.

Consider now the spatial polygon $\partial$ (St $A$ ), the boundary of the star of $A$. Draw in this polygon all the diagonals issuing from the vertex $p_{0}$ and also all diagonals joining the vertices $p_{i-1}$ and $p_{i+1}, 1 \leq i \leq m$; see Fig. 7 .

Let $D_{i}$ be the length of the diagonal from vertex $p_{0}$ to $p_{i}, 2 \leq i \leq m-1$, and let $d_{i}$ be the length of the diagonal joining vertices $p_{i-1}$ and $p_{i+1}, 1 \leq i \leq m, D_{2}=d_{1}$, $D_{m-1}=d_{m}$. We take for every five points $\left(A, p_{0}, p_{i-1}, p_{i}, p_{i+1}\right), 2 \leq i \leq m-1$, the corresponding Cayler-Menger equation:

$$
\alpha_{i} \bar{D}_{i}^{2}+\beta_{i} \bar{D}_{i}+\gamma_{i}=0
$$

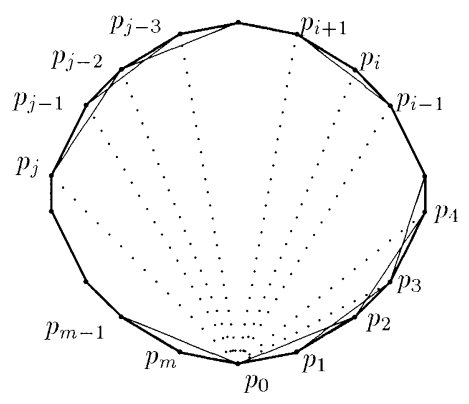

Fig. 7 
where $\alpha_{i}, \beta_{i}, \gamma_{i}$ must be written according to (6) with $x=\bar{D}_{i}, y=\bar{d}_{i}, p=\bar{D}_{i+1}$, $q=\bar{D}_{i-1}$ and $\bar{D}_{i}=D_{i}^{2}, \bar{d}_{i}=d_{i}^{2}$. In the case where we write (6) as a polynomial in $p$, we use (7), denoting the coefficients by the letters $f, g, h$ with subscripts corresponding to the number of $\left(18_{i}\right)$.

Now we eliminate $\bar{D}_{m-1}, \bar{D}_{m-2}, \ldots, \bar{D}_{3}$ from these equations successively. Start from $\bar{D}_{m-1}$. For this end consider two equations $\left(18_{m-1}\right)$ and $\left(18_{m-2}\right)$ written as follows:

$$
\begin{aligned}
\alpha_{m-1} & \bar{D}_{m-1}^{2}+\beta_{m-1} \bar{D}_{m-1}+\gamma_{m-1} \\
:= & \left(\bar{d}_{m-1}^{2}+a_{1}^{(m-1)} \bar{d}_{m-1}+b_{1}^{(m-1)}\right) \bar{D}_{m-1}^{2} \\
& +\left(a_{2}^{(m-1)} \bar{d}_{m-1}^{2}+b_{2}^{(m-1)} \bar{d}_{m-1} \bar{D}_{m-2}+b_{3}^{(m-1)} \bar{d}_{m-1}+b_{4}^{(m-1)} \bar{D}_{m-2}+b_{5}^{(m-1)}\right) \bar{D}_{m-1} \\
& +\left(a_{3}^{(m-1)} \bar{D}_{m-2}^{2}+b_{6}^{(m-1)} \bar{d}_{m-1}^{2}+a_{4}^{(m-1)} \bar{d}_{m-1} \bar{D}_{m-2}+b_{7}^{(m-1)} \bar{D}_{m-2}\right. \\
& \left.\quad+b_{8}^{(m-1)} \bar{d}_{m-1}+b_{9}^{(m-1)}\right)=0
\end{aligned}
$$

(we took into account that in our case $q=D_{m}$ is the length of the edge $\left\langle p_{0} p_{m}\right\rangle$ ),

$$
\begin{aligned}
& f_{m-2} \bar{D}_{m-1}^{2}+g_{m-2} \bar{D}_{m-1}+h_{m-2} \\
& :=a_{1}^{(m-2)} \bar{D}_{m-1}^{2}+\left[a_{2}^{(m-2)} d_{m-2} D_{m-3}+b_{1}^{(m-2)} \bar{d}_{m-2} \bar{D}_{m-2}+b_{2}^{(m-2)} \bar{D}_{m-3}\right. \\
& \left.\quad+b_{3}^{(m-2)} \bar{D}_{m-2}+b_{4}^{(m-2)} \bar{d}_{m-2}+b_{5}^{(m-2)}\right] \bar{D}_{m-1} \\
& \quad+\left[\bar{D}_{m-2}^{2} \bar{d}_{m-2}^{2}+a_{3}^{(m-2)} \bar{D}_{m-2}^{2} \bar{d}_{m-2}+a_{4}^{(m-2)} \bar{D}_{m-2} \bar{d}_{m-2}^{2}+a_{5}^{(m-2)} \bar{D}_{m-3}^{2}\right. \\
& \left.\quad+b_{6}^{(m-2)} \bar{d}_{m-2}^{2}+b_{7}^{(m-2)} \bar{D}_{m-2}^{2}+\text { the terms without the square of variables }\right] .
\end{aligned}
$$

The resultant of $\left(18_{m-1}\right)$ and $\left(18_{m-2}\right)$ as the determinant of a $4 \times 4$ matrix is the following:

$$
\left|\begin{array}{cccc}
\bar{d}_{m-1}^{2}+\cdots & a \bar{d}_{m-1}^{2}+\cdots & a \bar{D}_{m-2}^{2}+\cdots & 0 \\
0 & \bar{d}_{m-1}^{2}+\cdots & a \bar{d}_{m-1}^{2}+\cdots & a \bar{D}_{m-2}^{2}+\cdots \\
a_{1}^{(m-2)} & g_{m-2} & \bar{D}_{m-2}^{2} \bar{d}_{m-2}^{2}+a \bar{D}_{m-3}^{2}+\cdots & 0 \\
0 & a_{1}^{(m-2)} & \bar{d}_{m-2}\left(a \bar{D}_{m-3}+b \bar{D}_{m-2}\right)+\cdots & h_{m-2}
\end{array}\right|=0,
$$

where the element $g_{m-2}$ is equal to the written element $a_{43}$ and $h_{m-2}$ is equal to $a_{33}$ in the standard notations of matrix elements; for brevity we have also omitted the subscripts of some coefficients $a$.

Calculating this determinant, we obtain an equation of the following form:

$$
c_{m-2}^{(0)} \bar{D}_{m-2}^{4}+c_{m-2}^{(1)} \bar{D}_{m-2}^{3}+\cdots+c_{m-2}^{(4)} \bar{D}_{m-2}^{0}=0
$$

where $c$ (with super- and subscripts) are some polynomials in $\bar{D}_{m-3}, \bar{d}_{m-1}, \bar{d}_{m-2}$ with coefficients depending on $(l)$.

We study the structure of functions $c$ more thoroughly. The general $i$ th term $R_{i}$ of the resultant of $\left(18_{m-1}\right)$ and $\left(18_{m-2}\right)$ has the form

$$
R_{i}= \pm\left(\alpha_{m-1}\right)^{\mu_{0}^{(i)}}\left(\beta_{m-1}\right)^{\mu_{1}^{(i)}}\left(\gamma_{m-1}\right)^{\mu_{2}^{(i)}}\left(f_{m-2}\right)^{v_{0}^{(i)}}\left(g_{m-2}\right)^{v_{1}^{(i)}}\left(h_{m-2}\right)^{v_{2}^{(i)}}
$$

and, according to the theorem on the weight of the resultant's member, the following equalities on exponents $\mu$ and $\mu$ hold:

$\mu_{0}^{(i)}+\mu_{1}^{(i)}+\mu_{2}^{(i)}=2, \quad v_{0}^{(i)}+v_{1}^{(i)}+v_{2}^{(i)}=2, \quad \mu_{1}^{(i)}+2 \mu_{2}^{(i)}+v_{1}^{(i)}+2 v_{2}^{(i)}=4$. 
Substituting the values of $\alpha_{m-1}, \ldots, h_{m-2}$ in $R_{i}$ we obtain

$$
\begin{aligned}
R_{i}= & \pm a^{\nu_{0}}\left(\bar{d}_{m-1}^{2}+a \bar{d}_{m-1}+b\right)^{\mu_{0}}\left(a \bar{d}_{m-1}^{2}+b \bar{d}_{m-1} \bar{D}_{m-2}+\cdots\right)^{\mu_{1}} \\
& \times\left(a \bar{D}_{m-2}^{2}+b \bar{d}_{m-1} \bar{D}_{m-2}+b \bar{d}_{m-1}^{2}\right)^{\mu_{2}}\left(a \bar{d}_{m-2} \bar{D}_{m-3}+b \bar{d}_{m-2} \bar{D}_{m-2}+\cdots\right)^{\nu_{1}} \\
& \times\left(\bar{d}_{m-2}^{2} \bar{D}_{m-2}^{2}+a \bar{D}_{m-3}^{2}+\cdots\right)^{\nu_{2}}
\end{aligned}
$$

where for the sake of brevity we have omitted superscripts $(i)$ for $\mu$ and $\nu$ as well as the subscripts of the coefficients $a$ and $b$; moreover, in parentheses we have written only the greatest degrees of the corresponding variables.

Taking into account the conditions imposed on $\mu_{0}, \ldots, v_{2}$, by the formula for $R_{i}$, we come to the following conclusions: (1) in every $R_{i}$ the greatest degree of $\bar{D}_{m-2}$ is 4 ; (2) the greatest degree of $\bar{D}_{m-3}$ is 4 ; (3) the greatest total degree of $\bar{D}_{m-2}$ and $\bar{D}_{m-3}$ is 4 (indeed, the total degree of $\bar{D}_{m-2}$ and $\bar{D}_{m-3}$ in $R_{i}$ is $\mu_{1}+2 \mu_{2}+v_{1}+2 v_{2}=4$ ); (4) the greatest degree of $\bar{d}_{m-1}$ in $R_{i}$ is $2 \mu_{0}+2 \mu_{1}+2 \mu_{2}=2\left(\mu_{0}+\mu_{1}+\mu_{2}\right)=4$; (5) for the degrees of $\bar{d}_{m-2}$ we have $v_{1}+2 v_{2} \leq 4$ with equality to 4 iff $\mu_{1}=\mu_{2}=v_{1}=0, v_{2}=2$; (6) the greatest total degree of $\bar{d}_{m-1}$ and $\bar{d}_{m-2}$ is 8; and, finally, (7) the coefficient $c_{m-2}^{(0)}$ at $\bar{D}_{m-2}^{4}$ in (19) contains the monomial $\bar{d}_{m-1}^{4} \bar{d}_{m-2}^{4}$ with coefficient 1 . So we can present polynomial equation (19) in the following form:

$$
\begin{aligned}
& \bar{D}_{m-2}^{4}\left[\bar{d}_{m-2}^{4} \bar{d}_{m-1}^{4}+\cdots\left(\text { there is no degree of } \bar{D}_{m-3}\right)\right] \\
& +\bar{D}_{m-2}^{3}\left(b_{1} \bar{d}_{m-2}^{4} \bar{d}_{m-1}^{4}+\cdots(\text { maybe there is a term with the first }\right. \\
& \left.\left.\quad \text { degree of } \bar{D}_{m-3}\right)\right) \\
& +\cdots+\bar{D}_{m-2}^{-}\left(\bar{D}_{m-3}^{4}\left(b_{4} \bar{d}_{m-2}^{4} \bar{d}_{m-1}^{4}+\cdots\right)+\cdots\right)=0 .
\end{aligned}
$$

Now we can eliminate $\bar{D}_{m-2}$ from (20) and $\left(18_{m-3}\right)$, and so on. As the induction hypothesis, suppose that after $(k-1)$ eliminations we have come to the equation of the following form:

$\bar{D}_{m-k}^{T(k)}\left(\bar{d}_{m-k}^{T(k)} \cdots \bar{d}_{m-1}^{T(k)}+\cdots\right)+\cdots+\bar{D}_{m-k}^{0}\left[\bar{D}_{m-(k+1)}^{T(k)}\left(b \bar{d}_{m-k}^{T(k)} \cdots \bar{d}_{m-1}^{T(k)}+\cdots\right)+\cdots\right]=0$,

where $T(k)=2^{k}$, the greatest degree of $\bar{D}_{m-k}$ and $\bar{D}_{m-(k+1)}$ is $T(k)$, their greatest total degree is also $T(k)$, the greatest degree of each $\bar{d}_{i}, m-k \leq i \leq m-1$, is $T(k)$, the greatest total degree of $\bar{d}_{i}, m-k \leq i \leq m-1$, is $k T(k)$, and, finally, in the coefficient at $\bar{D}_{m-k}^{T(k)}$ the monomial $\bar{d}_{m-k}^{T(k)} \cdots \bar{d}_{m-1}^{T(k)}$ has the coefficient 1 . We show that after the elimination of $\bar{D}_{m-k}$ from (21) and $\left(18_{m-(k+1)}\right)$ we obtain a polynomial equation for $\bar{D}_{m-(k+1)}$ and $\bar{D}_{m-(k+2)}$ with properties similar to those of (21). Indeed, rewrite $(21)$ and $\left(18_{m-(k+1)}\right)$ as follows:

$$
\begin{gathered}
c_{0} \bar{D}_{m-k}^{T(k)}+c_{1} \bar{D}_{m-k}^{T(k)-1}+\cdots+c_{T(k)} \bar{D}_{m-k}^{0}=0 \\
f_{m-k-1} \bar{D}_{m-k}^{2}+g_{m-k-1} \bar{D}_{m-k}+h_{m-k-1} \\
:=a_{1}^{(m-k-1)} \bar{D}_{m-k}^{2}+\left[a_{2}^{(m-k-1)} \bar{d}_{m-(k+1)} \bar{D}_{m-(k+2)}+b_{1}^{(m-k-1)} \bar{d}_{m-(k+1)} \bar{D}_{m-(k+1)}\right. \\
+b_{2}^{(m-k-1)} \bar{D}_{m-(k+2)}+b_{3}^{(m-k-1)} \bar{D}_{m-(k+1)} \\
\left.+b_{4}^{(m-k-1)} \bar{d}_{m-(k+1)}+b_{5}^{(m-k-1)}\right] \bar{D}_{m-k}
\end{gathered}
$$




$$
\begin{aligned}
& +\left[\bar{D}_{m-(k+1)}^{2} \bar{d}_{m-(k+1)}^{2}+a_{3}^{(m-k-1)} \bar{D}_{m-(k+1)}^{2} \bar{d}_{m-(k+1)}\right. \\
& ++a_{4}^{(m-k-1)} \bar{D}_{m-(k+1)} \bar{d}_{m-(k+1)}^{2}+a_{5}^{(m-k-1)} \bar{D}_{m-(k+2)}^{2}+b_{6}^{(m-k-1)} \bar{d}_{m-(k+1)}^{2} \\
& \left.++b_{7}^{(m-k-1)} \bar{D}_{m-(k+1)}^{2}+(\text { terms without the square of variables) })\right]
\end{aligned}
$$

The general $i$ th term of the resultant of these equations is of the form

$$
\begin{gathered}
\pm c_{0}^{\mu_{0}} c_{1}^{\mu_{1}} \cdots c_{T(k)}^{\mu_{T(k)}}\left(a \bar{d}_{m-(k+1)} \bar{D}_{m-(k+2)}+b \bar{d}_{m-(k+1} \bar{D}_{m-(k+1)}+\cdots\right)^{\nu_{1}} \\
a^{\nu_{0}}\left(\bar{D}_{m-(k+1)}^{2} \bar{d}_{m-(k+1)}^{2}+a \bar{D}_{m-(k+1)}^{2} \bar{d}_{m-(k+1)}+a \bar{D}_{m-(k+1)} \bar{d}_{m-(k+1)}^{2}\right. \\
\left.\quad+a \bar{D}_{m-(k+2)}^{2}+b \bar{d}_{m-(k+1)}^{2}+b \bar{D}_{m-(k+1)}^{2}+\cdots\right)^{\nu_{2}}
\end{gathered}
$$

where for brevity we have omitted the superscript $(i)$ for $\mu$ and $v$ and also the subscripts of the coefficients $a$ and $b$; moreover, in parentheses we have written only the leading degrees of the corresponding variables. The $\mu$ and $v$ satisfy the equations:

$$
\begin{gathered}
\mu_{0}+\mu_{1}+\cdots+\mu_{T(k)}=2, \quad v_{0}+v_{1}+v_{2}=T(k), \\
\mu_{1}+2 \mu_{2}+\cdots+T(k) \mu_{T(k)}+v_{1}+2 v_{2}=2 T(k) .
\end{gathered}
$$

From these equations and (22) we can easily verify that the maximal degree of $\bar{D}_{m-(k+1)}$ in the resultant is $\mu_{1}+2 \mu_{2}+\cdots+T(k) \mu_{T(k)}+v_{1}+2 v_{2}=2 T(k)=T(k+1)$; the degree of $\bar{D}_{m-(k+2)}$ is $v_{1}+2 v_{2} \leq 2 T(k)$ with the equality for $v_{0}=0, v_{1}=0$, $v_{2}=T(k)$. Hence the greatest degree of $\bar{D}_{m-(k+2)}$ is $2 T(k)=T(k+1)$ too. The same is true for the greatest total degree of $\bar{D}_{m-(k+1)}$ and $\bar{D}_{m-(k+2)}$. Moreover, it is clear that the factor $\bar{d}_{m-(k+1)}^{2 T(k)}$ occurs only in the product $h_{m-k-1} \cdots h_{m-k-1}=h_{m-k-1}^{T(k)}$, so that in the coefficient at $\bar{D}_{m-(k+1)}^{T(k+1)}$ the monomial $\bar{d}_{m-(k+1)}^{T(k+1)} \bar{d}_{m-k}^{T(k+1)} \cdots \bar{d}_{m-1}^{T(k+1)}$ has coefficient 1 . Thus, it has been verified that the elimination process (by the induction hypothesis) after $(m-3)$ steps gives us an equation of the following form:

$$
\bar{D}_{2}^{T(m-2)}\left(\bar{d}_{2}^{T(m-2)} \bar{d}_{3}^{T(m-2)} \cdots \bar{d}_{m-1}^{T(m-2)}+\cdots\right)+\cdots+\bar{D}_{2}^{0} a\left(l, \bar{d}_{2}, \ldots, \bar{d}_{m-1}\right)=0,
$$

because $\bar{D}_{1}$ is the distance between $p_{0}$ and $p_{1}$ known as the length of the edge $\left\langle p_{0} p_{1}\right\rangle$. Therefore, in (23), all the coefficients depend only on $\bar{d}_{i}, 2 \leq i \leq m-1$, and $(l)$ including the last coefficient $a$ at $\bar{D}_{2}^{0}$. Since $\bar{D}_{2}=\bar{d}_{1}$, we can represent (23) as the equation for $\bar{d}_{1}, \bar{d}_{2}, \ldots, \bar{d}_{m-1}$ :

$$
\begin{gathered}
\bar{d}_{1}^{T(m-2)}\left(\bar{d}_{2}^{T(m-2)} \cdots \bar{d}_{m-1}^{T(m-2)}+\cdots\right)+\bar{d}_{1}^{T(m-2)-1}\left(b \bar{d}_{2}^{T(m-2)} \cdots \bar{d}_{m-1}^{T(m-2)}+\cdots\right) \\
+\cdots+\bar{d}_{1}^{0}\left(b \bar{d}_{2}^{T(m-2)} \cdots \bar{d}_{m-1}^{T(m-2)}+\cdots\right)=0
\end{gathered}
$$

where by $b$ we denote some coefficients (in general different) depending on the lengths of the edges. Equation (24) is the analog of (13) for a vertex of degree 4, and it reflects the fact that an $(m+1)$-hedral angle has $m-2$ degrees of freedom.

Now we use (24) for the successive elimination of variables $\bar{d}_{1}, \ldots, \bar{d}_{m-1}$. Let us make the first step. Consider equation $\left(17_{i}\right)$ for $i=1$ as a polynomial equation for $\bar{d}_{1}$ :

$$
\bar{d}_{1}^{K_{1}}\left(a_{1}(l) V^{2 M_{1}}+\cdots\right)+\cdots+\bar{d}_{1}^{0}\left(V^{4 N_{1}}+\cdots\right)=0,
$$


where $2 M_{1}<4 N_{1}$. For brevity, denote the exponent $T(m-2)$ by $T$ and the coefficient at $\bar{d}_{1}^{0}$ by the symbol “ $*$ " in (24). The resultant of $(24)$ and $\left(17_{1}^{\prime}\right)$ gives an equation of the following form:

$\left|\begin{array}{cccccc}\bar{d}_{2}^{T} \cdots \bar{d}_{m-1}^{T}+\cdots & b \bar{d}_{2}^{T} \cdots \bar{d}_{m-1}^{T}+\cdots & \cdots & * & \cdots & 0 \\ \vdots & \ddots & \vdots & \vdots & \vdots & \vdots \\ 0 & \cdots & \bar{d}_{2}^{T} \cdots \bar{d}_{m-1}^{T}+\cdots & \cdots & \cdots & * \\ a_{1}(l) V^{2 M_{1}}+\cdots & \cdots & \cdots & V^{4 N_{1}}+\cdots & \cdots & 0 \\ \vdots & \ddots & \vdots & \vdots & \ddots & \vdots \\ 0 & \cdots & a_{1}(l) V^{2 M_{1}}+\cdots & \cdots & \cdots & V^{4 N_{1}}+\cdots\end{array}\right|=0$,

which can be rewritten as follows (recall that in the resultant the first $K_{1}$ rows are composed by the coefficients of (24) and the last $T=T(m-2)$ ones are taken from $\left.\left(17_{1}^{\prime}\right)\right)$ :

$$
\begin{aligned}
\bar{d}_{2}^{L_{1}}\left(\bar{d}_{3}^{L_{1}} \cdots \bar{d}_{m-1}^{L_{1}}\left(V^{S_{1}}+\cdots\right)+\cdots\right) \\
\quad+\bar{d}_{2}^{L_{1}-1}\left(\bar{d}_{3}^{L_{1}} \cdots \bar{d}_{m-1}^{L_{1}}\left(b V^{S_{1}}+\cdots\right)+\cdots\right)+\cdots+\bar{d}_{2}^{0}(\cdots)=0,
\end{aligned}
$$

where $L_{1}=T(m-2) K_{1}$ and $S_{1}=4 T(m-2) N_{1}$ are the greatest degrees, respectively, of $\bar{d}_{i}, 2 \leq i \leq m-1$, and $V$. Now we can eliminate $\bar{d}_{2}$ using $\left(17_{2}^{\prime}\right)$ (which is $\left(17_{2}\right)$ rewritten for $\bar{d}_{2}=d_{2}^{2}$ ) and $\left(25_{1}\right)$. We obtain an equation which we can denote by $\left(25_{2}\right)$. Using this equation and its analogs, we can continue to eliminate $\bar{d}_{i}, i=3,4, \ldots$, by a similar procedure, and every time for $\bar{d}_{i}$ besides $\left(18_{i}^{\prime}\right)$ we have an equation (the analog of $\left.\left(25_{1}\right)\right)$ with the leading term $\bar{d}_{i}^{L_{i-1}} \bar{d}_{i+1}^{L_{i-1}} \cdots \bar{d}_{m-1}^{L_{i-1}} V^{S_{i-1}}$, where $L_{i-1}=L_{i-2} K_{i-1}=$ $T(m-2) K_{1} \cdots K_{i-1}, S_{i-1}=K_{i-1} S_{i-2}+4 N_{i-1} L_{i-2}$. Before the last step we have $\left(17_{m-1}^{\prime}\right)$, and an equation of the form

$$
\bar{d}_{m-1}^{L_{m-2}}\left(V^{S_{m-2}}+\cdots\right)+\bar{d}_{m-1}^{L_{m-2}-1}\left(b_{1} V^{S_{m-2}}+\cdots\right)+\cdots+d_{m-1}^{0}\left(b_{L_{m-2}} V^{S_{m-2}}+\cdots\right)=0,
$$

where $S_{m-2}$ is the greatest degree of $V$, and $b_{(\ldots)}$ are some coefficients polynomially depending on $(l)$. Hence it follows that their resultant gives the desired equation for $V$ :

$$
Q(V)=V^{2 N}+a_{1}(l) V^{2 N-2}+\cdots+a_{N}(l)=0,
$$

where $2 N=S_{m-2} K_{m-1}+4 N_{m-1} L_{m-2}$.

Remark. If we start the elimination of $\bar{D}_{i}$ from $\left(17_{j}\right)$ with some $j<m$ for $i=$ $j-1, j-2, \ldots, 1$, and, after obtaining an analog of (24), repeat the elimination of $\bar{d}_{i}$, we will finally obtain an equation relating $V$ and $D_{j}$.

Our arguments were valid under the assumption that the considered polyhedron $P$ is in a general position. Otherwise, by a small perturbation, we can transform $P$ to a near polyhedra $P_{\varepsilon}$ which is in a general position, and obtain for $P_{\varepsilon}$ the following equations: $Q_{\varepsilon}\left(V_{\varepsilon}\right)=0$. These equations are of the form (26) in which the polynomials $a_{i}\left(l_{\varepsilon}\right)$ have numerical coefficients independent of $\varepsilon$ (they depend only on the combinatorial structure $K$ of $P$ and on the choice of the vertex $A$ ). Taking the limit of $Q_{\varepsilon}$ as $\varepsilon \rightarrow 0$, we obtain for $V(P)$ the same equation (26). The lemma has been proved. 


\section{Proof of the Theorem}

Suppose first that the genus $g$ of polyhedra under consideration is zero. Suppose also that the theorem has been proved for all polyhedra of genus $g=0$ with the number of vertices at most $n$. Let $P$ be a polyhedron with $n+1$ vertices. If $P$ has an empty 3-cycle $G$, we can cut $P$ around $G$ and thereby obtain two polyhedra with boundary, both having less than $n+1$ vertices. Pasting their boundaries by triangular faces, we obtain two polyhedra $P_{1}$ and $P_{2}$ of genus $g=0$. Evidently, $V(P)=\varepsilon_{1}\left(P_{1}\right)+\varepsilon_{2} V\left(P_{2}\right)$, $\varepsilon_{i}= \pm 1$. By the induction hypothesis, both $P_{1}$ and $P_{2}$ have property $Q$, so it can be easily seen that the same is true for $P$ too.

If $P$ does not have an empty 3 -cycle, it has a combinatorial structure of type $K_{0}$ and, therefore, the lemma is applicable.

Suppose now that the theorem has been proved for all polyhedra of genus at most $g-1 \geq 0$. Let $P$ be an arbitrary polyhedron of genus $g$. Let $P_{0}$ be a polyhedron of genus $g$ with the minimum possible number of vertices. It is evident that $P_{0}$ necessarily has an empty 3-cycle $G$, for otherwise we could decrease the number of vertices. We cut $P_{0}$ around $G$ and paste two holes by triangular faces of opposite orientation. As a result, we obtain one or two polyhedra of genus less than $G$ which have property $Q$. Thus, the theorem is true for any polyhedron of genus $g$ with the minimum possible number of vertices. So for polyhedra of genus $g$ the base of induction is ready. Now we apply the lemma, if this is possible. Otherwise, the considered polyhedron $P$ has an empty 3 -cycle. Then, repeating the above argument, we obtain the desired result again. The theorem has been proved.

\section{Applications}

\subsection{The Isometric Realizations}

Suppose that $|K|$ is the carrier of a geometric simplicial 2-complex $K$ with given lengths of the edges. Then $|K|$ can be regarded as a 2-manifold with a prescribed polyhedral metric. One of the most important problems of metric geometry is the problem of isometric realization of $|K|$ as a polyhedral surface $P$ in $R^{3}$. There are many different settings of this problem; we require that the combinatorial structure of $K$ would be conserved, that is, $|K|$ must be a natural development of $P$. Up to the present time, the problem in such a general setting is far from being solved and there was no result of a positive or negative nature. Even the celebrated Alexandrov's theorem on the existence of an isometric realization of any convex polyhedral metric as a convex polyhedron in $R^{3}$ does not guarantee that $P$ will inherit the combinatorial structure of $K$. However, the main result of the present article immediately implies that for the existence of an isometric realization of $|K|$ in $R^{3}$ it is necessary that (5), originating from the combinatorial structure of $K$ with the given metric, has at least one root $V^{2} \geq 0$. Moreover, (5) gives us a priori all possible values of volume for the polyhedra sought, even without solving the existence problem. As to the construction of such polyhedra, we have designed [14] an algorithm which (in the generic case) gives a required polyhedron or otherwise establishes its nonexistence. 


\subsection{Volume as an Algebraic Number}

From (5) one can come to the trivial conclusion that if the lengths of the edges of a polyhedron are algebraic numbers, then its volume is an algebraic number too. The author believes that this observation could be a starting point for finding a deep explanation of the fact that the third Hilbert problem has an affirmative answer on the plane, where a polygon (even with integer values of lengths of the edges) may have a transcendental value of the area. Meanwhile, in space the answer is negative; recall that this problem is concerned with the question of equipartity of the two polytopes having equal volumes.

\section{Acknowledgment}

In conclusion I would like to thank my anonymous referees for their useful critical remarks and my colleagues A. M. Stepin, Z. G. Psiola, and S. Lawrencenko for their help in improving the English of this article.

\section{References}

1. Alexander, R. Lipschitzian mappings and total mean curvature of polyhedral surfaces, I, Trans. Amer. Math. Soc., 288(2) (1985), 661-678.

2. Alexandrov, V. An example of a flexible polyhedron with nonconstant volume in the spherical space, Beitr. Algebra Geom., 38(1) (1997), 11-18.

3. Astrelin, A. V., and Sabitov, I. Kh. Minimal degree polynomial for the volume calculation of an octahedron by its metric, Uspekhi Mat. Nauk, 50(4) (1995), 245-246.

4. Berger, M. Geometrie, vol. 2, 2nd edn., Cedic/Fernand Nanhan, PA, 1979.

5. Blumental, L. M. Theory and Applications of Distance Geometry, 2nd edn., Chelsea, NY, 1970.

6. Connelly, R. The rigidity of polyhedral surfaces, Math. Mag., 52(5) (1978), 275-283.

7. Connelly, R. Conjectures and open questions in rigidity, Proc. Internat. Congr. Math., Helsinki, 1978, vol. 1, pp. 407-414.

8. Connelly, R., Sabitov, I., and Walz, A. The Bellows conjecture, Beitr. Algebra Geom.. 38(1) (1997), 1-10.

9. Ivanova-Karatopraklieva, I., and Sabitov, I. Kh. Bending of surfaces, Part II, J. Math. Sci., 74(3) (1995), 997-1043.

10. Pavlova, O. V. Suspension's volume as a function of length of its edges, Uspekhi Mat. Nauk, 50(4) (1995), 251-252.

11. Sabitov, I. Kh. The local theory of bendings of surfaces (translated from Russian) in Encyclopedia of Mathematical Sciences, vol. 48, Springer-Verlag, Berlin, pp. 179-250.

12. Sabitov, I. Kh. On the problem of volume invariance for flexing polyhedra, Uspekhi Mat. Nauk, 50(2) (1995), 223-224.

13. Sabitov, I. Kh. The volume of polyhedron as a function of its metric, Fund. Prikl. Mat., 2(4) (1996), 1235-1246.

14. Sabitov, I. Kh. The volume of polyhedron as a function of its metric and algorithmical solution of the main problems in the metric theory of polyhedra, Internat. School-Seminar Devoted to the N. V. Efimov's Memory (1996), Abstracts, Rostov University, pp. 64-65.

Received September 23, 1996, and in revised form June 10, 1997, and November 6, 1997. 